\title{
Cryptogenic organising pneumonia
}

\section{To the Editors:}

We have read with interest the paper by CORDIER [1] describing the current understanding of the pathogenesis, aetiology, diagnosis and treatment of cryptogenic organising pneumonia (COP). The author acknowledged that corticosteroids remain the standard treatment of COP, usually resulting in rapid clinical, and slower radiological, improvements. Although the response to corticosteroids is generally good, a proportion of patients can experience early or late relapses. In addition, some patients may be unable to tolerate corticosteroids, or the disease may continue to deteriorate, despite therapy with corticosteroids. Therefore, there is a need for alternative therapies to treat this condition. CORDIER [1] only briefly mentioned the occasional improvement of COP to treatment with antibiotics, especially macrolides [2].

Macrolides have gained increasing use in the management of diffuse panbronchiolitis, asthma and cystic fibrosis [3]. It has been proposed that the activity of macrolides in these conditions is through anti-inflammatory, as well as antimicrobial, action. In vitro studies suggest that macrolides may have an immunomodulatory activity, exhibited through: 1) the inhibition of the pulmonary influx of neutrophils; 2) the reduction of inflammatory cytokines, such as interleukin-8; 3) the protection of the epithelium from bioactive phospholipids; and 4) the improvement of the transportability of airway secretions [3]. Some of these activities may affect the underlying mechanisms involved in the pathogenesis of COP, therefore explaining a potential role of macrolides as therapeutic agents to treat this condition.

Recently, there has been increasing evidence of the role of macrolides in the treatment of COP. For example, STOVER et al. [4] described their experience using macrolides to treat three patients with COP and three patients with radiation-induced bronchiolitis obliterans organising pneumonia. Over the last few years we have also gained experience in the use of macrolides in the treatment of COP. To date, we have treated three patients with clinical and/or histological confirmation of COP. Two of our patients presented with an acute illness and one had a more gradual presentation. All three patients received clarithromycin $500 \mathrm{mg}$ b.i.d. and the duration of therapy ranged between 3-12 months. All of the patients showed improvement in their symptoms, together with the radiological resolution of pulmonary infiltrates and improvement in inflammatory markers. One of the patients was originally treated with oral corticosteroids but failed to improve, however, once macrolides were introduced his condition improved and within a few weeks the corticosteroids were able to be withdrawn.

Based on our observations, and the report by STOVER et al. [4], we believe that the duration of macrolide therapy should be between 3-12 months, although caution should be exhibited. The decision to treat cryptogenic organising pneumonia patients with macrolides should, at present, be made on an individual basis with the proviso that if improvement is not observed, or if a patient's condition worsens, conventional therapy with corticosteroids should be introduced. Macrolides could be considered for use in combination with corticosteroids in patients who fail to respond to corticosteroids or as a corticosteroid sparing agent. We would suggest wider reporting of experience to gain more information on the role of macrolides in cryptogenic organising pneumonia.

\section{J.A. Kastelik, M. Greenstone, D.V. McGivern and A.H. Morice}

Dept of Respiratory Medicine, Castle Hill Hospital, Cottingham, Hull, East Yorkshire HU16 5JQ, UK.

\section{REFERENCES}

1 Cordier J-F. Cryptogenic organising pneumonia. Eur Respir J 2006; 28: 422-446.

2 Epler GR, Colby TV, McLoud TC, Carrington CB, Gaensler FA. Bronchiolitis obliterans organizing pneumonia. N Engl J Med 1985; 312: 152-158.

3 Rubin BK, Henke MO. Immunomodulatory activity and effectiveness of macrolides in chronic airway disease. Chest 2004; 125: Suppl. 2, 70S-78S.

4 Stover DE, Mangino D. Macrolides: a treatment alternative for bronchiolitis obliterans organizing pneumonia? Chest 2005; 128: 3611-3617. 